\title{
Syphla-Chek: a Qualitative Study
}

\author{
PATRICIA E. DZIUK, DEBORAH A. BLACK, AND BRADFORD L. THERRELL, JR.* \\ Clinical Chemistry Branch, Bureau of Laboratories, Texas Department of Health Resources, Austin, \\ Texas 78756
}

Received for publication 15 December 1976

\begin{abstract}
A qualitative evaluation of the newly marketed Syphla-Chek Test (Hyland, Div. of Travenol Laboratories, Inc., Costa Mesa, Calif.), a macroscopically read, nontreponemal card test, was undertaken in order to further evaluate the validity of this procedure as an aid in the diagnosis of syphilis. A total of 1,074 randomly selected sera submitted to the Texas Department of Health Resources for routine analysis, for confirmational analysis, and as a result of problems in analysis, were examined using the Venereal Disease Research Laboratory (VDRL) slide test, the Rapid Plasma Reagin (circle) Card Test (RPRCT), SyphlaChek Test, and the fluorescent treponemal antibody absorption (FTA-ABS) test. Comparison of the reagin testing procedures studied versus the FTA-ABS procedure led to the following order of agreement with FTA-ABS reactive results: Syphla-Chek, 88.8\%; RPRCT, 87.1\%; and VDRL, 83.2\%. Agreement with FTA-ABS nonreactive results was in the order: RPRCT, 86.7\%; Syphla-Chek, 79.6\%; and VDRL, 78.7\%. The relatively few minor procedural problems encountered with the Syphla-Chek Test during this study are also discussed.
\end{abstract}

The Hyland Division of Travenol Laboratories, Inc. (Costa Mesa, Calif.), has recently marketed Syphla-Chek, a new, nontreponemal, macroscopic slide test for syphilis. This test, like the Rapid Plasma Reagin (circle) Card Test (RPRCT; Hynson, Westcott, and Dunning, Baltimore, Md.; 6-8) and the Reagin Screen Test (RST; Lederle Laboratories, Pearl River, N.Y.; $1,2)$, relies on macroscopic agglutination of a specially prepared cardiolipin antigen (5) as a positive indication of the presence of reagin (indicative of syphilitic infection). In the case of Syphla-Chek, the antigen has been complexed with kaolin and choline chloride, using thimerosal as a preservative. The test procedure employs a transparent slide viewed against a black background and relies on formation of light-colored granules as an indication of reactivity.

Literature supplied with the Syphla-Chek kit reports that 1,471 serological specimens were compared for reactivity with the Venereal Disease Research Laboratory (VDRL) test. The specificity of Syphla-Chek in this comparison appeared comparable to that of the VDRL. Similarly, sensitivity studies on 414 patients with confirmed diagnosis of primary, secondary, or latent syphilis showed almost identical agreement with the VDRL procedure. We have undertaken a comparative qualitative evaluation of the Syphla-Chek test in order to further investigate the ease of use and reliability of this procedure under normal conditions in our laboratory.

\section{MATERIALS AND METHODS}

Syphla-Chek test kit. Syphla-Chek Test kits (Hyland 050-023) were supplied by the manufacturer for evaluation. Each kit contained five 3-ml ampules of antigen, one 3.3-ml bottle of positive human control serum, one 3.3-ml bottle of negative human control serum, a dispenser vial with a calibrated needle, 75 10-well plastic reaction slides, and a sufficient supply of plastic pipettes for $\mathbf{7 5 0}$ determinations. Additional equipment necessary in the laboratory included a mechanical rotator capable of $180 \mathrm{rpm}$, Pasteur pipettes for transferring antigen from ampule to dispensing vial, mixing sticks (flat toothpicks), nonreflecting black background (construction paper), humidifying cover (optional in dry areas), light source (fluorescent desk lamp), and a refrigerator capable of maintaining storage temperatures between 2 and $8^{\circ} \mathrm{C}$.

Specimens. All specimens were sera obtained from the Medical Serology Branch, Bureau of Laboratories, Texas Department of Health Resources. The serum specimens studied were randomly selected from routine specimens received in the laboratory. Most nonreactive specimens were submitted from local venereal disease clinics, whereas most reactive specimens were received as confirmatory or problem specimens. Special care was taken to insure that all selected sera were less than $72 \mathrm{~h}$ old at the time of analysis as specified in the Syphla-Chek package insert. (It is recommended that specimens not analyzed within $72 \mathrm{~h}$ after collection be frozen at $-20^{\circ} \mathrm{C}$ until analyzed.)

The sera studied were heat inactivated (a condition not required in either the Syphla-Chek or RPRCT procedure) and analyzed in the Medial Serology Branch using standard VDRL and fluorescent treponemal antibody absorption (FTA-ABS) test 
techniques (8). Syphla-Chek and RPRCT results were obtained independently on each of the coded specimens, with care taken at all times to insure complete adherence to information contained in the package insert.

Method. Initially, in order to become familiar with the new testing procedure, several groups of sera were analyzed as a training exercise for the technologists involved. The investigators evaluating the test were selected from their demonstrated competency in reading macroscopic card tests, having previously read well over 100,000 RPRCTs and several hundred RSTs. Both had little trouble in adjusting to the Syphla-Chek procedure. During the preliminary training, Syphla-Chek readings were compared closely with VDRL and FTA-ABS test results, especially in instances requiring subjective determinations, until both technologists were both competent at and confident of all procedural techniques.

All analyses were performed exactly as outlined in the package insert (November 1975). The qualitative test methodology includes dispensing $0.05 \mathrm{ml}$ of serum into a transparent sample well in one of the plastic slides provided. One drop $(1 / 60 \mathrm{ml})$ of antigen is added from the reagent dispenser supplied, and the contents of the well are mixed with a mixing stick while spreading the specimen about the entire well. The slide is rotated at $180 \mathrm{rev}$ for $6 \mathrm{~min}$, followed by macroscopic examination against a black background using reflected lighting (diagram of correct reading technique given in package insert). Reactive specimens are characterized by white agglutinate masses in contrast to smooth granular suspensions in the case of nonreactive specimens. Both a positive and a negative human control are included as a part of the kit.

For our qualitative study, 1,074 serum specimens were examined by both Syphla-Chek and RPRCT (14 $\mathrm{mm}$ ) procedures (6) for comparison with the VDRL and FTA-ABS results routinely obtained. Specimens exhibiting a borderline FTA-ABS result were not included in the testing comparison. The results of all comparative testings are included in Tables 1 to 4 .

\section{RESULTS AND DISCUSSION}

The total tabulation of results from all of the tests, Syphla-Chek, RPRCT (14 mm), VDRL, and FTA-ABS, is listed in Table 1. Various calculations are possible from these data. In view of the unavailability of clinical histories on routine specimens received in this laboratory, and because the FTA-ABS procedure has been demonstrated to be both sensitive and specific in diagnosing syphilis $(3,4)$, FTA-ABS results were used as a basis for comparing the reactivity of each of the reagin tests studied. Qualitative comparisons between each of the tests performed and the FTA-ABS results are listed in Tables 2 through 4.

Relative agreements between the reagin tests studied and the FTA-ABS reactive results varied as follows: Syphla-Chek, 88.8\%; RPRCT, 87.1\%; and VDRL, 83.2\%. Agreements with FTA-ABS nonreactive results were: RPRCT, 86.7\%; Syphla-Chek, 79.6\%; and VDRL, 78.7\%. Agreements between the various reagin tests were $93.9 \%$ between Syphla-Chek and RPRCT, $89.2 \%$ between VDRL and Syphla-Chek, and

TABLE 2. Qualitative comparison of Syphla-Chek and FTA-ABS ${ }^{a}$

\begin{tabular}{lcccc}
\hline \multicolumn{1}{c}{ Test } & \multicolumn{2}{c}{ Agreement } & \multicolumn{2}{c}{ Disagreement } \\
\hline Syphla-Chek & $\mathbf{R}$ & $\mathbf{N}$ & $\mathbf{R}$ & $\mathbf{N}$ \\
FTA-ABS & $\mathbf{R}$ & $\mathbf{N}$ & $\mathbf{N}$ & $\mathbf{R}$ \\
Total & & & & \\
& 545 & 366 & 94 & 69 \\
\hline
\end{tabular}

a $N$, Nonreactive; $R$, reactive.

TABLE 3. Qualitative comparison of RPRCT and FTA-ABS ${ }^{a}$

\begin{tabular}{llccc}
\hline \multicolumn{1}{c}{ Test } & \multicolumn{2}{c}{ Agreement } & \multicolumn{2}{c}{ Disagreement } \\
\hline RPRCT & $\mathbf{R}$ & $\mathbf{N}$ & $\mathbf{R}$ & $\mathbf{N}$ \\
FTA-ABS & $\mathbf{R}$ & $\mathbf{N}$ & $\mathbf{N}$ & $\mathbf{R}$ \\
Total & 535 & 399 & 61 & 79 \\
\hline
\end{tabular}

a $N$, Nonreactive; $R$, reactive.

TABLE 4. Qualitative comparison of VDRL and FTA-ABS ${ }^{a}$

\begin{tabular}{lllcc}
\hline \multicolumn{1}{c}{ Test } & \multicolumn{2}{c}{ Agreement } & \multicolumn{2}{c}{ Disagreement } \\
\hline VDRL & $\mathbf{R}$ & $\mathbf{N}$ & $\mathbf{R}$ & $\mathbf{N}$ \\
FTA-ABS & $\mathbf{R}$ & $\mathbf{N}$ & $\mathbf{N}$ & $\mathbf{R}$ \\
Total & 511 & 360 & 100 & 103 \\
\hline
\end{tabular}

a $N$, Nonreactive; $R$, reactive.

TABLE 1. Tabulation of qualitative testing results

\begin{tabular}{lcccccccc}
\hline \multicolumn{1}{c}{ Test } & \multicolumn{10}{c}{ Classification of test result } \\
\hline Syphla-Chek & $\mathbf{R}$ & $\mathbf{R}$ & $\mathbf{R}$ & $\mathbf{R}$ & $\mathbf{N}$ & $\mathbf{N}$ & $\mathbf{N}$ & $\mathbf{N}$ \\
RPRCT & $\mathbf{R}$ & $\mathbf{N}$ & $\mathbf{R}$ & $\mathbf{N}$ & $\mathbf{R}$ & $\mathbf{N}$ & $\mathbf{R}$ & $\mathbf{N}$ \\
VDRL & $\mathbf{R}$ & $\mathbf{N}$ & $\mathbf{N}$ & $\mathbf{R}$ & $\mathbf{R}$ & $\mathbf{N}$ & $\mathbf{N}$ & $\mathbf{R}$ \\
FTA-ABS & $\mathbf{R ~ N}$ & $\mathbf{R} \mathbf{N}$ & $\mathbf{R} \mathbf{N}$ & $\mathbf{R} \mathbf{N}$ & $\mathbf{R} \mathbf{N}$ & $\mathbf{R} \mathbf{N}$ & $\mathbf{R} \mathbf{N}$ & $\mathbf{R} \mathbf{N}$ \\
Totals & 48055 & $\mathbf{7 1 5}$ & 464 & 1220 & $\mathbf{5} 1$ & 46340 & 41 & 1424 \\
\hline
\end{tabular}

\footnotetext{
a $\mathrm{N}$, Nonreactive; $\mathrm{R}$, reactive.
} 
88.4\% between the VDRL and the RPRCT. Total agreements (positive and negative) between the FTA-ABS procedure and the other test procedures studied were: RPRCT, 87.0\%; Syphla-Chek, 84.8\%; and VDRL, 81.1\%. These values compare favorably with results obtained in our previous investigation of another macroscopic card test (1) where reagin test agreement with the FTA-ABS procedure was $85.5 \%$ for the RPRCT and $79.8 \%$ for the VDRL.

We noted essentially no discrepancies in the reading of results between technologists or in readings on randomly repeated specimens when checking for consistency of interpretation. Although no major procedural problems were encountered, reading the test results presented slight difficulties due to the necessity of reflecting light from a source onto a nonglaring black background and back through the transparent sample well. At the manufacturers recommendation, fluorescent lighting was employed, and after each technologist had established the best angle of reflection for her, lighting ceased to be a problem. Lint adhering to the back of the transparent well plate also caused a problem during reading, as did moisture condensation. Passing the card across a clean, lintfree cloth prior to reading generally eliminated either of these problems. Additionally, rotation at $180 \mathrm{rpm}$ caused the sample slides to be thrown from the rotator unless they were weighted, taped, placed in a holder, or placed under a humidifying cover. And, as with other tests of this sort, use of a commercial micropipetter rather than the disposable pipettes provided seemed to be a more reliable method of dispensing reproducible serum samples.

Packaging of the Syphla-Chek Test kit ( $\mathrm{Hy}$ land, Div. of Travenol Laboratories, Inc.) provides the necessary materials for performing 750 determinations. While similar in many respects to the RPRCT kits (Hynson, Westcott, and Dunning) presently in wide use, the $\mathrm{Hy}$ land kit, as does the recently marketed RST kit (Lederle Laboratories), contains vials of reactive and nonreactive control sera not presently included in RPRCT kits. The Hyland kit does not contain a weakly reactive control, which is included in the Lederle kit, but does use human sera as opposed to rabbit sera used in the RST kit. The Syphla-Chek kit packaging is slightly deficient in that the analytical procedure calls for mixing the serum specimen and antigen about the specimen well, although it provides no mixing sticks (toothpicks will suffice in this respect).

Although the Syphla-Chek Test offers no substantial advantages over other nontreponemal card tests, it does appear to have merit as an alternative for those not satisfied with present techniques. From our study of a specimen population including $57 \%$ reactive FTA-ABS sera from patients on whom clinical histories were not available, the Syphla-Chek Test appears to possess satisfactory reactivity as a qualitative test to justify its acceptance as a standard test for syphilis. As with all nontreponemal procedures, users must remember that "false positive" reactions may occur due to any of a number of causes, and a confirmatory procedure continues to be necessary prior to final diagnosis.

\section{ACKNOWLEDGMENTS}

We wish to express our appreciation to Marcia McBride, Hortence Grace, and Mary Rutter of the Medical Serology Branch, Bureau of Laboratories of the Texas Department of Health Resources, for their assistance in performing VDRL and FTA-ABS procedures on the specimens used in this study. Additionally, acknowledgment is given to Charles Sweet, Chief, Bureau of Laboratories, and again to Marcia McBride for their helpful discussions during the course of these investigations, and to Beverly Ihlo, Rebecca Stephens, and Cynthia Casas for their help in preparing the manuscript.

\section{LITERATURE CITED}

1. Black, D. A., P. E. Ray, and B. L. Therrell. 1976. Qualitative evaluation of the Reagin screen test. J. Clin. Microbiol. 4:16-18.

2. Dyckman, J. D., R. D. Wende, D. Gantenbein, and R. P. Williams. 1976. Evaluation of Reagin screen, new serological test for syphilis. J. Clin. Microbiol. 4:145150.

3. Hunter, E. F., W. E. Deacon, and P. E. Meyer. 1964. An improved FTA test for syphilis, the absorption procedure (FTA-ABS). Public Health Rep. 79:410 412.

4. Mackey, D. M., E. V. Price, J. M. Knox, and A. Scotti. 1969. Specificity of the FTA-ABS test for syphilis. J. Am. Med. Assoc. 207:1683-1685.

5. Pangborn, M. C., J. O. Almeida, F. Maltaner, A. M. Silverstein, and W. R. Thompson. 1955. Cardiolipin antigens. WHO Monogr. Ser. 6.

6. Portnoy, J. 1963. Modifications of the rapid plasma Reagin (RPR) card test for syphilis, for use in largescale testing. Am. J. Clin. Pathol. 40:473-479.

7. Portnoy, J., J. H. Brewer, and A. Harris. 1962. Rapid plasma Reagin card test for syphilis and other treponematoses. Public Health Rep. 77:645-652.

8. United States Public Health Service. 1969. Manual of tests for syphilis. Public Health Service publication no. 411. U.S. Government Printing Office, Washington, D.C. 